\title{
Deficiência Intelectual e Saúde Mental: Quando a Fronteira Vira Território
}

Intellectual Disability And Mental Health: When The Border Land Becomes

Discapacidad Intelectual Y Salud Mental: Cuando La Frontera Se Convierte En Territorio

\section{Luciana Togni de Lima e Silva Surjus}

Universidade de Campinas, São Paulo, SP, Brasil.

\section{Rosana Teresa Onocko Campos}

Universidade de Campinas, São Paulo, SP, Brasil.

\begin{abstract}
Resumo:
O presente artigo propõe o destaque da interface entre os campos Deficiência Intelectual e Saúde Mental, ainda subestimado no Brasil, mas que vem se constituindo internacionalmente como objeto de investigações dada a prevalência do chamado Diagnóstico Dual, qual seja, a significativa ocorrência de problemas de saúde mental nas pessoas com DI. Situando-se na perspectiva da pesquisa qualitativa e participativa, a partir da Hermenêutica Filosófica, resgataram-se as tradições teóricas constitutivas desses dois campos, SM e DI, bem como a recente produção sobre suas intersecções e, a partir do diálogo dos achados entre a revisão da literatura e as experiências de profissionais e gestores de serviços de atenção psicossocial, buscou-se compreender como tem se dado esta interface no cotidiano dos serviços, a fim de contribuir com a qualificação das ações e da construção de Políticas Públicas. Análises preliminares apontam a relevância da problematização da temática para a superação da histórica institucionalização das pessoas com DI.
\end{abstract}

Palavras chave: Deficiência intelectual, Saúde mental, Diagnóstico dual, Política pública

\begin{abstract}
This article aims to highlight the interface between the intellectual disabilities and the mental health fields, still underestimated in Brazil but increasingly becoming an object of scientific study around the world due to the prevalence of the dual diagnosis, it means, intellectual disability (ID) and mental illness co-occurring. Using qualitative research methods and participatory approaches through the philosophy hermeneutic, considering the traditional theories which lay the foundation of these two fields, mental illness and intellectual disability, and also the recent literature on the intersections between them and from the experiences of psychosocial assistance center workers and directors, it intends to understand how these interfaces has been
\end{abstract}


happened in the everyday of health services, in order to qualify for acting on these places and also building Public Policies. Preliminary analysis indicates the relevancy of problematize this theme contributing to overcome the historical psychiatric institutionalization of this population. Key-words: Intellectual disability; Mental health; Dual diagnosis; Philosophy hermeneutic; Public polices

\section{Resumen}

Este artículo se propone a resaltar la interfaz entre los campos de la Discapacidad Intelectual y la Salud Mental, aún subestimado en Brasil, pero que se constituye internacionalmente como objeto de investigación dada la prevalencia del Diagnóstico Dual, es decir, la importante aparición de problemas de salud mental en las personas con DI. Centrándose en la perspectiva de la investigación cualitativa y participativa, a partir de la hermenéutica filosófica, se rescataron las tradiciones teóricas constitutivas de estos dos campos, SM y DI, así como la reciente producción de sus intersecciones y, a partir del dialogo de los resultados entre la revisión de la literatura y las experiencias de profesionales y gestores de servicios de atención psicosocial, se buscó la comprensión acerca de como se da dicha interfaz en el cotidiano de los servicios, con el objetivo de contribuir a la calificación de las acciones y la construcción de políticas públicas. Los análisis preliminares indican la importancia de problematizar la tematica para la superación de la institucionalización histórica de las personas con DI.

Palabras clave: Discapacidad intelectual, Salud mental, Patología dual, la Política pública

\section{E da experiência nascem as indagações}

S, 24 anos, chegou ao CAPS acompanhada da irmã, para submeter-se a uma triagem. Foi encaminhada ao serviço após ter seu pedido de internação recusado em um hospital psiquiátrico, efeito da reformulação da assistência em Saúde Mental da cidade de Campinas. S. tinha em sua história uma única tentativa (frustrada) de vinculação a uma entidade para pessoas com deficiência intelectual, e dezenas de internações; tendo, na última delas, sido abandonada pelo pai. A irmã, sensibilizada, levou-a para sua casa, mas já não conseguia negociar com marido e filha, a perturbadora presença de S. em suas vidas. Durante os minutos em que aguardava no CAPS, sentada no chão, emitia grunhidos, rasgava revistas e sujava de fezes as paredes do banheiro... A triagem ainda não havia sido realizada, mas o veredito já havia sido dado por profissionais e usuários: ali não era o seu lugar... 
Após vivenciar intensamente os desafios da reorganização do modelo de assistência em Saúde Mental na cidade de Campinas-SP, atuando como profissional, gestora local e pesquisadora; vivenciando ainda a gestão de entidade que apoia pessoas com deficiência intelectual, surge uma questão: em contextos que avançaram na Reforma Psiquiátrica, qual é o lugar que tem sido possibilitado (e com qual intenção) às pessoas com importantes limitações cognitivas e significativas repercussões subjetivas?

Como uma demanda inadequada aos serviços de saúde mental, aparentemente paralela, mas com certa constância, pessoas com deficiência intelectual (PCDI) chegam até a saúde mental. Quase nunca com queixas próprias, senão inferidas por outrem, levantando timidamente as questões: estariam apresentando sintomas? Seriam alterações de comportamento próprias à deficiência? Estariam sem a assistência adequada? Teriam associado algum transtorno mental? Estariam ainda esquecidas por detrás dos muros dos manicômios?

Esses dois campos, deficiência intelectual (DI) e saúde mental (SM), apesar de compartilharem da mesma origem histórica, situados lado a lado na luta pela defesa dos direitos humanos, com sua população foco enclausurada pelos mesmos muros, acabaram por seguir rumos próprios. A SM, constituída sob os preceitos da desinstitucionalização e superação do modelo hospitalocêntrico, vem se firmando através da políticas pública de saúde, a partir de importantes reformulações no campo conceitual, clínico e ético-político (Amarante, 2000) e viabilizando-se a partir de um aparato legal que orienta a reorganização de uma rede de serviços ${ }^{1}$ sensíveis a sua demanda clínica, que inclui no espectro de sua responsabilidade, ações de âmbito comunitário e social.

No Brasil, apesar do longo caminho percorrido, e talvez somente a partir dele, percebe-se que ainda há muito a se construir na articulação de políticas intersetoriais - não por acaso, tema da IV Conferência Nacional de Saúde Mental de 2010. Para a sustentação de processos efetivos de inclusão social certamente urge a extrapolação do campo da saúde para superar alguns desafios: para além dos Serviços Residenciais Terapêuticos, favorecer que a as pessoas com transtornos mentais graves consiga acessar a política pública de habitação; ampliar o acesso e a sustentação da permanência nos espaços de educação - tanto das crianças, como de jovens e adultos; estabelecer parcerias efetivas com a rede serviços sócio assistenciais; ampliar iniciativas de geração de renda e economia solidária, bem como avaliar novas possibilidades de produzir a emancipação dos usuários e fomentar relações mais solidárias no trabalho; am- 
pliar e resgatar o movimento de organização de usuários e famílias.

$\mathrm{Na}$ Pesquisa Avaliativa dos CAPS de Campinas-SP (Onocko Campos, 2008; Surjus, 2007; Figueiredo et al, 2008) evidenciou-se a exclusão dos usuários ao que se refere ao acesso a direitos fundamentais. Dos usuários em acompanhamento nos CAPS naquele momento, 53\% não tinham concluído o ensino fundamental, $65 \%$ não estavam inseridos em nenhuma modalidade do mercado de trabalho e, $86 \%$ tinham fonte de renda vinculada a aposentadoria, auxílio doença ou a benefício previdenciário. Os referidos estudos não investigaram as condições de moradia dos usuários. Outro achado ainda da referida pesquisa foi o reconhecimento nos CAPS de alto percentual de PCDI, inseridas a partir de comorbidades psiquiátricas e/ou da profunda falta de outras ofertas mais adequadas.

Todavia, a expansão da rede de CAPS, "carro chefe" da mudança de paradigma da política de saúde mental, recebe o mandato, na prática, de articular todas as demandas no campo da saúde mental. Ademais, apesar de não reconhecidas como demanda adequada à estruturação dos serviços, as PCDI já configuravam naquele momento o terceiro maior percentual da população atendida, por categorias diagnósticas, perfazendo um total de $8 \%$ (Surjus, 2007; Figueiredo et al, 2008).
Voltando-nos para o campo político e teórico constitutivo da DI reconhecemo-lo oriundo de recentes reformulações conceituais, incluindo da própria nomenclatura (Sassaki, 2005). Oficialmente utilizado em 1995 pela ONU, o termo Deficiência Intelectual tem sido preferencialmente utilizado haja vista a maior apropriação, referindo-se ao funcionamento do intelecto especificamente e também com o objetivo de diferenciação dos transtornos mentais, delimitando como diferentes territórios e reforçando a deficiência como condição que deve preferencialmente ser abordada a partir da verificação de apoios necessários e de transformações de seu entorno, para garantir sua inclusão social.

A noção de incapacidade tem sido amplamente reconhecida como um produto social, o que ganha perspectiva na Classificação Internacional de Funcionalidade, Incapacidade e Saúde (CIF), publicada pela Organização Mundial de Saúde (Who, 2001), instrumento complementar à CID10, que, para além de uma perspectiva médica, inclui a societária e ambiental.

\footnotetext{
Anteriormente también se entendía por discapacidad intelectual la falta de habilidades para funcionar en el día a día y que su origen estaba en el bajo funcionamiento intelectual. Hoy la concepción es más amplia, entendiendo que la discapacidad intelectual indica la existencia de áreas en las que la persona necesita apoyos. Así, si
} 
una persona tiene dificultades para sumar, se le puede prestar el apoyo de una calculadora y así mejorar su funcionamiento. Esta nueva visión hace que la discapacidad intelectual no sea un diagnóstico determinista, sino una puerta para ofrecer los apoyos necesarios y mejorar así la calidad de vida. (Mateos, 2003, p.13)

A área da DI, portanto, constituiu-se a partir da luta das pessoas com deficiência, num esforço para superação do modelo médico e na busca pela operacionalização de um modelo biopsicossocial, que considere as pessoas com deficiência dotadas de capacidades e incapacidades e da necessidade da provisão de apoios que garantam sua participação social. Tal prerrogativa é legitimada pela Convenção Internacional dos Direitos das Pessoas com Deficiência (Resende \& Vital, 2008), que ganha no Brasil força de emenda constitucional.

Segundo dados do censo populacional de 2010, estima-se que quase 3 milhões de brasileiros (1,4\% da população) tenham deficiência intelectual, sendo seu referenciamento assistencial quase que totalmente restrito a instituições de caráter filantrópico sendo inegável o protagonismo das mesmas nos avanços de diferentes políticas públicas. Podemos citar como exemplo a reestruturação da política de educação - inicialmente especial e mais recentemente inclusiva; na legislação trabalhista através da Lei de Rev. Polis e Psique, 2013; 3(2):82-96
Cotas (Brasil,1991) no mercado formal de trabalho, o direito ao atendimento preferencial (Brasil, 2000), a garantia de benefícios previdenciários e a serviços específicos da assistência social (CNAS, 2009), como Centros Dia e Residências Inclusivas. Percebe-se, porém, que não houve na mesma dimensão avanços na formulação da política pública de saúde, gerando uma ideia falseada de um campo "inclusivo".

A prática cotidiana dos serviços mobilizava a seguinte questão: será que a separação histórica entre as redes assistenciais em Saúde Mental e em Deficiência intelectual, acabou por minimizar ou mesmo negligenciar necessidades de saúde desta população?

Apesar do reconhecimento de inúmeras dificuldades para se estabelecer critérios fidedignos para estudos epidemiológicos sobre a prevalência e incidência dos transtornos mentais em PCDI, a literatura internacional estima que cerca de $40 \%$ das mesmas têm associados diagnósticos de transtornos mentais e/ou comportamentais (Salvador et al., 2000; Cowley, 2004; Cooper et al., 2007; Smiley, 2007; Martorell et al., 2008; Fletcher, 2009).

Não se trata aqui de negar a especificidade dos fenômenos em questão, nem de reconduzir a deficiência intelectual ao estatuto de doença, mas de reconhecer que o desafio da inclusão social depende também de 
garantir-lhes o direito à saúde e à prevenção de agravos, e que o impacto dos problemas de SM podem reduzir significativamente a possibilidade de inclusão social das PCDI, questão que talvez estejamos subestimando.

Desta forma, o objetivo deste estudo foi investigar a relevância da problematização da DI no campo da SM por meio da revisão da literatura relativa ao tema, do mapeamento da rede de serviços de referência para PCDI e da compreensão de como profissionais e gestores dos Centros de Atenção Psicossocial concebem esta interface nos seus serviços. Esperamos assim, contribuir para a formulação e qualificação de políticas públicas vigentes.

\section{Como fizemos}

Para Gadamer (1997) mais do que uma metodologia, a Hermenêutica é uma postura filosófica. Um modo de construção de conhecimento que reconhece a historicidade do pesquisador, e o destaque do objeto de estudo a partir da fusão de horizontes passado-presente - na busca pelas respostas a questões atuais, não compreendidas. Neste perspectiva, o momento do destaque do objeto - sem recorrer ao habitual "recorte", poderia possibilitar sua compreensão sem negar seu contexto de produção (Onocko Campos \& Furtado, 2006), além de conter seu próprio princípio de aplicação.
De acordo com o autor, as questões emergem de uma história efeitual - história que faz efeito no pesquisador histórico, a partir da qual o objeto se destaca, e produz-se sentido a partir de recorrer às tradições que se ocuparam de semelhantes questões. Encontramos, portanto, no recurso às tradições a possibilidade de efetivação do círculo de compreensão hermenêutico, no qual pré-concepções podem então ser revisitadas, e o objeto destacado não se distancia das suas condições de produção (Onocko Campos \& Furtado, 2006).

Nosso esforço hermenêutico, portanto, inicia-se a partir de nosso reconhecimento de pertença à tradição da Saúde Mental pública brasileira, e da transformação de nossas pré-concepções - o único ponto de partida possível para compreensão em Gadamer - em questões. Ao refletir a importância das tradições, Gadamer (1997) nos alerta ao fato de que as tradições falam sempre em múltiplas vozes, o que nos imporia o desafio de estarmos atentos às vozes mais fracas, silenciadas e apagadas na história (Onocko Campos \& Furtado, 2008). Não obstante mover-se por uma questão inicial, o retorno às tradições que se preocuparam com questões semelhantes deveria em nosso caso, portanto, considerar e fazer dialogar as produções científicas atuais, construções de outros setores considerados "não científicos", 
e a experiência de quem cotidianamente se envolve com a temática em questão.

A pesquisa tomou como campo os Municípios que dispõem de Centros de Atenção Psicossocial tipo III (funcionamento 24 horas) no Estado de São Paulo. Ressalta-se que São Paulo é a unidade federativa com o maior número de CAPS III no Brasil, o que consiste que o campo abarcou mais de um terço dos serviços deste tipo no país.

O encontro proposto efetivou-se a partir da realização de um Curso de Avaliação de Serviços de Saúde Mental, sendo a Deficiência Intelectual um dos temas propostos para a problematização e construção de indicadores de avaliação dos CAPS. A revisão da literatura foi apresentada a partir de exposição sobre o tema, posteriormente colocada em diálogo com o compartilhamento de experiência entre os participantes a respeito da temática.

Sendo um dos eixos priorizados no curso realizado, o tema da DI foi tratado a partir da metodologia proposta, incluindo atividade de imersão, que consistia na identificação da rede de atenção às PCDI na região de abrangência dos serviços, a visitação de uma das instituições e a realização de análise crítica sobre a inserção de PCDI nos CAPS. Tal atividade produziu um mapeamento preliminar acerca das instituições Rev. Polis e Psique, 2013; 3(2):82-96 designadas para atendimento, nas regiões envolvidas no curso.

\section{O que colhemos no caminho}

Cabe ressaltar a dificuldade em mobilizar os alunos do curso em se empenharem especialmente com atividade relacionada a esta temática, numa postura inicial de estranhamento e distanciamento desta perspectiva em sua prática. Entretanto, percebeu-se que a estratégia da composição entre atividades de dispersão, atualização teórica e análise participativa, produziram uma primeira desterritorialização nas certezas de que o tema não era questão para saúde mental.

O mapeamento das instituições explicita uma oferta de serviços composta em sua totalidade por instituições de caráter filantrópico e pouco variada. Dos 43 serviços identificados em 28 cidades, 25 são Associações de Pais e Amigos dos Excepcionais (APAE); duas outras associações semelhantes, mas referentes ao Autismo e Síndrome de Down; seis são escolas especializadas; uma desenvolve exclusivamente ações para inclusão no mercado formal de trabalho. As demais se referem à acolhimento institucional ou instituições de longa permanência, como no caso da única instituição pública presente no presente mapeamento.

O compartilhamento dessa experiência de ida à campo, se deu a partir da análise 
coletiva e participativa dos achados, e evidenciou a lacuna de diretrizes políticas para o atendimento a esta população, gerando um cenário fragmentado, de poucas articulações e intercâmbios, bem como a ausência de serviços públicos. A questão dos problemas de saúde mental foi por vezes apontada como critério de exclusão ao acesso a algumas das instituições identificadas, e fator de inúmeras dúvidas em relação a sua inclusão nos serviços de saúde mental.

A descrição dos casos que chegam aos CAPS evidencia que muitas das PCDI tem a internação em Hospitais Psiquiátricos como primeira oferta no campo da saúde mental, invertendo todo o histórico processo da Reforma Psiquiátrica, onde a internação é recurso último a ser acionado, e prioritariamente em Hospitais Gerais. Mais recentemente, alguns referenciamentos derivam de encaminhamentos a partir dos CAPS infantojuvenis.

Quanto aos principais diagnósticos que levam ao encaminhamento aos CAPS, são apontados quadros psicóticos e transtornos de personalidade, em sua maioria já em uso de psicofármacos associados. Os profissionais relatam inúmeras situações de agravamento como a falta total de suporte para as famílias, a completa exclusão das possibilidades de convívio social, recorrência de abuso e alta vulnerabilidade social, culmi- nando em comportamentos de agressividade intensa e hipersexualidade.

Muitas são as dificuldades descritas pelos profissionais no que se refere ao atendimento das PCDI nos CAPS. Não há consenso sobre o entendimento deste serviço como referência para esta população, tendo em muitas situações o diagnóstico da DI como fator impeditivo ao reconhecimento do adoecimento psíquico. Antes mesmo do acolhimento à demanda, é comum que a recepção dessa população nos CAPS se restrinja aos esforços para identificar uma instituição especializada para onde encaminhar. Entretanto, impera o consenso do risco iminente à institucionalização quando esta população adentra os Hospitais Psiquiátricos.

O manejo adequado apresenta-se como um desafio, reconhecendo certa limitação na compreensão para além das características estereotipadas de uma pessoa com DI, ou para além de sintomas. A sobreposição dos diagnósticos parece encobrir quase que totalmente o sujeito que deveria/poderia emergir.

Fez-se presente nestas discussões o fato de que muitos dos usuários que permanecem no CAPS nos espaços de convívio, sem grandes investimentos para construção de Projetos Terapêuticos Singulares (PTS) (Brasil, 2008), são pessoas com DI. Não parece ser presente nos espaços de discussão da equipe, esta problematização. 
Da mesma forma, a condição "dual" parece produzir nos serviços estratégias ora confusas, ora desresponsabilizadas: a disponibilidade de ambas as Redes, tanto para PCDI, quanto para as questões de SM, parece se fechar quando da ocorrência dos dois fenômenos simultaneamente. Entre as situações descritas, revela-se muitas vezes o desligamento da instituição de referência para pessoas com DI quando eclode o adoecimento psíquico.

Já nos CAPS - todos eles referência para população prioritariamente de adultos, mantém-se a expectativa de que a parceria com as instituições para pessoas com DI reproduzissem o já superado "ciclo fisioterapia/terapia ocupacional/fonoaudiologia" ao longo de toda a vida, tecnologias que comumente compõem as abordagens de estimulação precoce, e posteriormente empregadas diante de necessidades específicas.

\section{Perspectivas}

Inúmeras dúvidas puderam fazer transitar as questões sobre o que o CAPS poderia ofertar - entre as tecnologias disponíveis e as necessárias criações para corresponder ao desconhecido, alcançando certa possibilidade de refletir sobre o que as pessoas com DI poderiam oferecer para a construção de seus próprios PTS. Revisitando as práticas psicossociais, muitos profissio- nais puderam reconhecer que as abordagens junto às pessoas com DI aproximam-se dos pressupostos de uma clínica de domínio dos mesmos, operando para produzir ampliação dos coeficientes de autonomia (Onocko Campos \& Campos, 2006), com vistas à inclusão social.

A discussão sistemática dos casos em reuniões multiprofissionais e interdisciplinares, a organização de equipes de referência, e o recurso do apoio matricial especializado (Campos, 1999; Campos \& Domitti, 2007; Brasil, 2008) entre diferentes redes e a composição de Projetos Terapêuticos Singulares intersetoriais, que compõem o desafio cotidiano nestes serviços, se ressignificam na especificidade de cada um que ali se insere.

A própria função dos CAPS é convocada às discussões em sua concepção de serviço articulador de redes de atenção numa lógica substitutiva ao modelo asilar, e sua dificuldade na superação de tomar "casos" para si. Neste aspecto, a lacuna assistencial de serviços de referência para DI, como também a articulação frágil entre SM e serviços existentes aumenta a insegurança dos CAPS em fazer frente às demandas, integralmente e sem parceiros. Desta forma, externa-se a preocupação de que o CAPS não reproduza cronificação, respondendo ao mandato social de encarcerar a diferença. 
Foram apresentadas por alguns serviços estratégias que são lançadas no sentido da aproximação para melhor qualificação das necessidades e das respostas a elas. Grupos específicos para pessoas com DI nos CAPS, avaliações dos ganhos relacionais a partir da inserção no serviço, o cuidado às famílias e a problematização do que se espera como melhora, tem efetivado um novo olhar, mais inclusivo, que reconhece demandas legítimas e intervenções efetivas. Esta mudança de postura tem possibilitado, ao revés, começar a contar também com as instituições para DI na composição de projetos de egressos de Hospitais Psiquiátricos, moradores dos SRT, prática anteriormente sequer considerada.

A exemplo da Política Nacional de Saúde da Pessoa Portadora de Deficiência, instituída pela Portaria GM/MS n. ${ }^{\circ} 1060$, de 5 de junho de 2002, que preconiza a promoção da qualidade de vida das pessoas portadoras de defíciência e a assistência integral à saúde, entre outros princípios, começam a ser descritas algumas experiências exitosas de atendimento de pessoas com DI nas Unidades Básicas de Saúde, e a potência dos Centros de Convivência ao se abrir para toda a comunidade, em se constituir como uma importante porta de acesso à saúde para populações em situação de vulnerabilidade.

No tocante qualificação da assistência às PCDI atendidas nos CAPS, pro- posições surgiram no sentido de promover atenção especial para os casos de DI que chegam aos serviços, cuidado e prevenção ao prejuízo cognitivo dos usuários dos CAPS, e a necessária articulação com a rede de atenção em DI.

De acordo com o caminho metodológico proposto, faz-se necessário revisitar as questões que motivaram a produção deste artigo, concluindo provisoriamente algumas inquietações que, certamente produzem novas questões.

Parece-nos neste momento mais seguro afirmar que, embora persistindo muitos entraves no processo de diagnóstico de TM, as PCDI convivem com o acúmulo de fatores de risco ao desenvolvimento deste adoecimento. Todas as vozes tomadas pela pesquisa parecem convergir para os limites aos processos de inclusão das PCDI, agravados pela ocorrência de TM, o que se traduz muitas vezes em pela negligência de suporte dos serviços tanto da rede de apoio a pessoa com deficiência quanto da rede de saúde mental.

No Brasil, embora coexistam políticas públicas dos dois campos em questão, parece não ocorrer a problematização a respeito dos problemas de saúde mental da população com DD em nenhuma delas, ainda que dois censos de moradores de hospitais Psiquiátricos alertam ao possível e silencioso destino (Gomes et al., 2002; Barros \& Bichaff, 2008). 
Cabe evidenciar, contudo, esforços recentes na organização do Sistema Único de Saúde (SUS) a partir do estabelecimento de Redes de Atenção, normatizadas pelo decreto presidencial 7508 de 2012, e reafirmadas no Plano Nacional de Saúde (2011 a 2014), que traz como desafio a ampliação do acesso e da qualidade da atenção em todo o SUS. Também parece historicamente oportuna a proposição, dentre as redes priorizadas em sua implantação, a presença da Rede de Atenção Psicossocial (Brasil, 2011) e de Cuidados à Pessoa com Deficiência (Brasil, 2012).

Reafirmamos, portanto, a necessidade de abertura de pontos de diálogo entre gestores e profissionais das Redes em questão, sensibilizando-as para a construção de qualificação do acesso e da atenção às PCDI, como também de ações preventivas. Silenciar essa problematização pode significar a ampliação da histórica segregação como resposta primeira aos cenários de desassistência e negligência.

\section{Nota}

1 Incluem-se aqui os CAPS, os Serviços Residenciais Terapêuticos, os Centros de Convivência e iniciativas de Geração de Renda, os Núcleos de Apoio à Saúde da Família, Leitos de Saúde Mental em Hospital Geral, etc.

\section{Referências}

Barros, S. \& Bichaff, R. (orgs.). (2008) Desafios para a desinstitucionalização: censo psicossocial dos moradores em hospitais psiquiátricos do Estado de São Paulo/São Paulo: FUNDAP. Secretaria da Saúde.

Brasil. Lei 8.213 de 24 de julho de 1991. Dispõe sobre os Planos de Benefícios da Previdência Social e dá outras providências. Disponível em: $\quad<$ http://www.planalto.gov.br/ ccivil 03/leis/18213cons.htm $>$. Data de acesso: 04 de maio de 2013.

Brasil. Lei 10.048 de 8 de novembro de 2000. Dá prioridade de atendimento às pessoas que especifica, e dá outras providências. Disponível em: $<\underline{\text { http:// }}$ www.planalto.gov.br/ccivil_03/leis/ 110048.htm>. Data de acesso: 04 de maio de 2013.

Brasil. Ministério da Saúde. (2008) Secretaria-Executiva. Núcleo Técnico da Política Nacional de Humanização. Clínica ampliada, equipe de referência e projeto terapêutico singular. Brasília: Ministério da Saúde.

Brasil. Portaria 3.088, de 23 de dezembro de 2011. Institui a Rede de Atenção Psicossocial para pessoas com 
sofrimento ou transtorno mental e com necessidades decorrentes do uso de crack, álcool e outras drogas, no âmbito do Sistema Único de Saúde. Disponível em: <http:// www.brasilsus.com.br/legislacoes/ gm/111276-3088.html>. Data de acesso: 04 de maio de 2013.

Brasil. Portaria $\mathrm{n}^{\mathrm{o}} 793$, de 24 de abril de 2012. Institui a rede de cuidados à pessoa com deficiência no âmbito do sistema único de saúde. Disponível em: $<$ http://bvsms.saude. gov.br/bvs/saudelegis/gm/2012/ prt0793_24_04_2012.html>. Data de acesso: 04 de maio de 2013.

Campos, G. W. de S. (1999) Equipes de referência e apoio especializado matricial: um ensaio sobre a reorganização do trabalho em saúde. Ciência e Saúde Coletiva, Rio de Janeiro, v. 4, n. 2, p. 393-403.

Campos G. W. de S.; Domitti, A. C. (2007) Apoio matricial e equipe de referência: uma metodologia para gestão do trabalho interdisciplinar em saúde. Caderno de Saúde Pública, Rio de Janeiro, v. 23, n. 2, p. $399-407$.

Conselho Nacional De Assistência Social. Resolução 109 de 2009. Aprova a Tipificação Nacional de Serviços Socioassistenciais. Disponível em: $\quad<\underline{\text { http://www.mds.gov.br/ }}$ assistenciasocial/protecaobasica/ cras/documentos/Tipificacao $\% 20$ Nacional $\% 20 \mathrm{de} \% 20$ Servicos $\% 20$ Socioassistenciais.pdf $>$. Data de acesso: 04 de maio de 2013.

Cooper, S et al. (2007) Mental ill-health in adults with intellectual disabilities: prevalence and associated factors. British Journal of Psychiatry, 190: 27-35.

Cowley, A et al. Descriptive psycopathology in people with mental retardation. Journal of Nervous and Mental Disease, 2004; 192:232-237.

Figueiredo, M. D et al.. (2008) Uma Análise Complementar do Perfil dos CAPS do Município de Campinas. In: Onocko Campos, R et al. Pesquisa Avaliativa em Saúde Mental: desenho participativo e efeitos da narratividade. São Paulo: Aderaldo \& Rothschild.

Fletcher, R. J. (2009) Clinical Usefulness of Diagnostic Manual-Intellectual Disability for Mental Disorders in Persons With Intellectual Disability: Results From a Brief Field Survey. Journal Clin Psychiatry, 70:0, Month.

Furtado, J. P. (2006) Avaliação da situação atual dos Serviços Residenciais Terapêuticos no SUS. Ciência \& 
Saúde Coletiva, Rio de Janeiro, vol. 11, n. 3, p. 785-795.

Furtado, J. P. \& Onocko Campos, R. T. (2008) O percurso da pesquisa avaliativa de uma rede de Centros de Atenção Psicossocial: entre a saúde coletiva e a saúde mental. In: Onocko Campos, R. T et al. Pesquisa avaliativa em saúde mental: desenho participativo e efeitos de narratividade. São Paulo: Aderaldo \& Rothschild, p.189-208.

Gomes, M. P. C et al. (2002) Censo dos pacientes internados em uma instituição asilar no Estado do Rio de Janeiro: dados preliminares. Cad. Saúde Pública, Rio de Janeiro, 18(6):1803-1807, nov-dez.

Instituto Brasileiro De Geografia E Estatística. Disponível em: < $\underline{\text { http:// }}$ www.ibge.gov.br/home/presidencia/ noticias/noticia_visualiza.php?id $\underline{\text { noticia }=438 \& \text { id pagina }=1 \text {, em } 17 \mathrm{de}}$

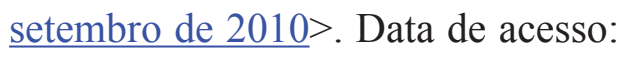
04 de maio de 2013.

MARTORELL, A et al. (2008) Identification of personal factors that determine work outcome for adults with intellectual disability. Journal of Intellectual Disability Reserch, 52 (pt 12): 1091-1101.

Mateos, J. L. A et al. (2003) Discapacidad Intelectual y Salud Mental - guía práctica. FEAPS (Confederación Rev. Polis e Psique, 2013; 3(2):82-96
Española de Organizaciones a favor de las personas com DI): Madri-ES. Moss, S. et al. (1997) Validity of the PASADD for detecting psychiatric symptoms in adults with learning disability (mental retardation). Social Psychiatry and Psychiatric Epidemiology: 32, p. 344-354.

Onocko Campos, R \& Campos G. S. W. (2006) Co-construção de autonomia: o sujeito em questão. In: Campos et al. Tratado de saúde coletiva. São Paulo - Rio de Janeiro: HucitecFiocruz, pp. 669-88.

Onocko Campos, R et al. (2008) Pesquisa Avaliativa em Saúde Mental Desenho participativo e efeitos da narratividade. São Paulo: Editora Hucitec.

Resende, A. P. C. \& Vital, F. M. de P. (coord.). (2008) A Convenção sobre Direitos das Pessoas com Deficiência Comentada - Secretaria Especial dos Direitos Humanos. Coordenadoria Nacional para Integração da Pessoa Portadora de Deficiência, Brasília. Inclui a íntegra da Convenção sobre Direitos das Pessoas com Deficiência e o Protocolo Facultativo e a Declaração Universal dos Direitos Humanos.

Salvador-Carulla, L et al. (2000) Hidden psychiatric morbidity in a vocational programme for people with 
intellectual disability. Journal of Intellectual Disability Reserch; 44 (Pt 2):147-154.

Sassaki, R. K. (2005) Atualizações semânticas na inclusão de pessoas: Deficiência mental ou intelectual? Doença mental ou transtorno mental? Revista Nacional de Reabilitação, ano IX, n. 43, p.9-10.

Smiley E. et al. (2007) Incidence and predictors of mental ill-health in adults with intellectual disabilities. Prospective study. The British Journal of Psychiatry, 191: 313-319.

Surjus, L. T. L. S. (2007) Narrativas Políticas: o olhar dos usuários sobre os CAPS de Campinas. 133p. Dissertação (mestrado em Saúde Coletiva). Universidade Estadual de Campinas.

Who World Health Organization. (2001) Internacional Classification of functioning, disability and health: ICF. World Health Organization.

\section{Luciana Togni de Lima e Silva Surjus:} Doutoranda em Saúde Coletiva pela UNICAMP. Mestre em Saúde Coletiva pela e UNICAMP (2005). Especialista em Saúde Pública pela UNICAMP (2002). Graduada em Terapia Ocupacional pela PUCCampinas (1999). Tem experiência clínica, de gestão e supervisão na área de Saúde Coletiva, com ênfase em Saúde Mental e Deficiência Intelectual. Integrante do Grupo de
Pesquisa (CNPq) Saúde Coletiva e Saúde Mental: Interfaces\&quot; do Departamento de Saúde Coletiva da Faculdade de Ciências Médicas UNICAMP. Desenvolve pesquisa de Avaliação Participativa de serviços de saúde mental, tendo como temática do Doutorado a interface entre o campo da saúde mental e deficiência intelectual. Atualmente Coordena o processo de reorientação da Rede de Atenção Psicossocial de Sorocaba/SP.

E-mail: lucianatogni@hotmail.com

Rosana Teresa Onocko Campos: Possui graduação em Ciências Médicas pela Universidade Nacional de Rosário (1986), mestrado em Saúde Coletiva pela Universidade Estadual de Campinas (1998), doutorado em Saúde Coletiva pela Universidade Estadual de Campinas (2001) e livre-docência pela Universidade Estadual de Campinas (2013). Desde 2004 é professor RDIDP da Universidade Estadual de Campinas e coordena o Programa de Pós-graduação em Saúde Coletiva da Faculdade de Ciências Médicas. Tem experiência na área de Saúde Coletiva, com ênfase nos seguintes temas: saúde coletiva, gestão e subjetividade, saúde mental, planejamento em saúde e políticas públicas, avaliação de políticas e serviços. Foi assessor da Política Nacional de Humanização em seus primórdios e da Coordenação Nacional de Saúde Mental/MS. Participa ativamente da formação de médicos e é Supervisora dos Programas de Aprimoramento em Saúde Mental e em Planejamento e administração 
de serviços de saúde, desde 1998. Coordena o grupo de pesquisa Saúde Coletiva e saúde mental: Interfaces desde 2003. É responsável pela \&quot;Pesquisa Avaliativa de Saúde Mental: indicadores para avaliação e monitoramento dos CAPS III do estado de São Paulo\&quot; (finanaciamento FAPESP) e coordena o projeto Alliance de Recherche
Université et Communauté ARUC no Brasil (financiamento IDRC). Além disso, é orientadora de mestrado e doutorado. Bolsista PQ $1 \mathrm{C}$ do CNPq.

E-mail: rosanaoc@mpc.com.br 